\title{
A ténue linha entre arquitetura e escultura: "The sculpture village" (1987), Frank Gehry e Anthony Caro
}

\author{
Pasqualino Grosso \\ paki.gro@me.com \\ Arquiteto, Itália \\ Carola Brandini \\ brandinicarola@libero.it \\ Arquiteta, Itália
}

Para citação: GROSSO, Pasqualino; BRANDINI, Carola - A ténue linha entre arquitetura e escultura: "The sculpture village" (1987), Frank Gehry e Anthony Caro. Estudo Prévio 18. Lisboa: CEACT/UAL - Centro de Estudos de Arquitetura, Cidade e Território da Universidade Autónoma de Lisboa, 2020, p. 28-36. ISSN: 2182-4339 [Disponível em: www.estudoprevio.net]. DOI: https://doi.org/10.26619/2182-4339/18.4

Artigo recebido a 1 de outubro de 2020 e aceite para publicação a 13 de dezembro de 2020 . Creative Commons, licença CC BY-4.0: https://creativecommons.org/licenses/by/4.0/

\section{Resumo}

Frank Gehry (Toronto, 1929) um dos mais debatidos arquitetos contemporâneos, é considerado um dos expoentes máximos do desconstrutivismo arquitetónico. Trabalha a decomposição dos edifícios em diferentes unidades volumétricas, recorrendo para isso à combinação de materiais pouco comuns - como rede metálica, chapa ondulada ou mesmo complexas ligas à base de titânio -, e a práticas que remetem para as técnicas do collage artístico. Partindo destas premissas, procura-se neste artigo refletir acerca da sua abordagem da relação entre arquitetura e escultura, a partir de um projeto pouco conhecido, o Sculpture Village, realizado em 1987 em colaboração com artistas de renome mundial - entre os quais Anthony Caro e Jon Isherwood -, no quadro de um workshop organizado pela rede internacional de artistas Triangle Artists em 1987. Neste projeto, que acabou por ser demolido pouco tempo depois da sua conclusão, a escultura é protagonista, mesmo se concebida em termos arquitetónicos. Assim, através de entrevistas, textos e fotografias fornecidas diretamente pelos participantes e pelos organizadores do evento acerca deste projeto, os autores propõem uma reflexão acerca da relação entre escultura e arquitetura que possa lançar pistas para uma mais abrangente leitura do trabalho de Gehry.

Palavras-Chave: Arquitetura, Escultura, Frank Gehry, Anthony Caro, Triangle Artists 


\title{
The fine line between architecture and sculpture: The sculpture village (1987), Frank Gehry and Anthony Caro
}

\begin{abstract}
Frank Gehry (Toronto, 1929) is considered one of the key figures of architectural deconstructivism and is one of the most discussed contemporary architects. Above all, he developed an interest in the process of building's decomposition into different volumetric units, made using the combination of different and unusual materials - from the metal mesh, to the corrugated sheet, up to complex titanium-based alloys - and according to practices that recall the technique of artistic collage. With these statements, this article we tried to understand his approach towards the relationship between architecture and sculpture starting from an almost unknown project, the Sculpture Village, built in 1987 in collaboration with worldwide known artists, including Anthony Caro, Jon Isherwood, in the frame of a workshop promoted by the international network of artists Triangle Artists. In this project, demolished shortly after it was completed, sculpture is the protagonist, conceived in architectural terms. Through interviews, texts and photographs provided directly by participants and founders of the project, the authors started an in-depth analysis on the relationship between sculpture and architecture and a broader understanding of Gehry's work.
\end{abstract}

Palavras-Chave: Architecture, Sculpture, Frank Gehry, Anthony Caro, Triangle Artists

\section{Triangle Artists}

O projeto Sculpture Village nasce em 1987, na sequência do encontro entre o arquiteto Frank Gehry e os artistas britânicos Anthony Caro e John Isherwood, seu assistente. Foi construído no espaço de duas semanas, durante um workshop promovido na Mashomack Fish and Game Preserve, Pine Plains, junto a Nova York, pelo coletivo de artistas Triangle Artists, de que Caro era fundador, juntamente com Sheila Girling, sua mulher. O nome Triangle Artists surge na sequência do workshop inaugural, em 1982, para o qual Caro tinha convidado dez artistas provenientes de três países diferentes: Grã-Bretanha, Canadá e Estados Unidos. Apesar ter sido pensado como um evento isolado, o workshop acabou por se repetir, em edições regulares, dada a enorme adesão, ao longo dos anos, por parte dos $\operatorname{artistas}^{1}$ que encontravam neste projeto características únicas que o diferenciavam de qualquer outra das iniciativas do género realizadas à época. $\mathrm{O}$ objetivo fundamental era proporcionar momentos de intensa discussão e partilha, e a maior parte dos artistas não experimentava este tipo de vivência desde o tempo de estudantes. ${ }^{2}$

"In 25 years, the model has proved so popular that it has spread from New York - where it started in 1982 in rural Dutchess County before migrating first to the World Trade Center and then to DUMBO - all over the world. Workshops have taken place in locations from Cape Town, South Africa, to Kunming, China, to a fishing village in Martinique." (Taylor, 2007) 


\section{estudoprévio}

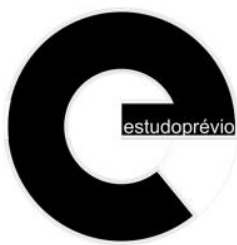

PT | P02 | EP18| w2021

Em 1987, pela primeira e última vez, houve arquitetos a participar no workshop, seguindo uma sugestão e com o apoio de Lord Palumbo, colecionador de arte e grande apaixonado por arquitetura moderna e contemporânea. De facto, aquele que foi o principal impulsionador da iniciativa, ainda disponibilizou uma elevada verba para o desenvolvimento do projeto, sendo que parte deste montante serviu, precisamente, para financiar a vinda dos arquitetos de renome internacional convidados a participar nesta edição do workshop.

Os pintores e os escultores, organizados em vários grupos, trabalharam, assim, com a dupla de arquitetos Alison e Peter Smithson, do Reino Unido, e com os arquitetos americanos William McDonough e Frank Gehry. A Anthony Caro, coube-lhe trabalhar com Frank Gehry e outros dois arquitetos do seu atelier - Susan Nardulli e Paul Lubowicki -, fazendo também parte da equipa os escultores Jon Isherwood e Sheila Girling. ${ }^{3}$

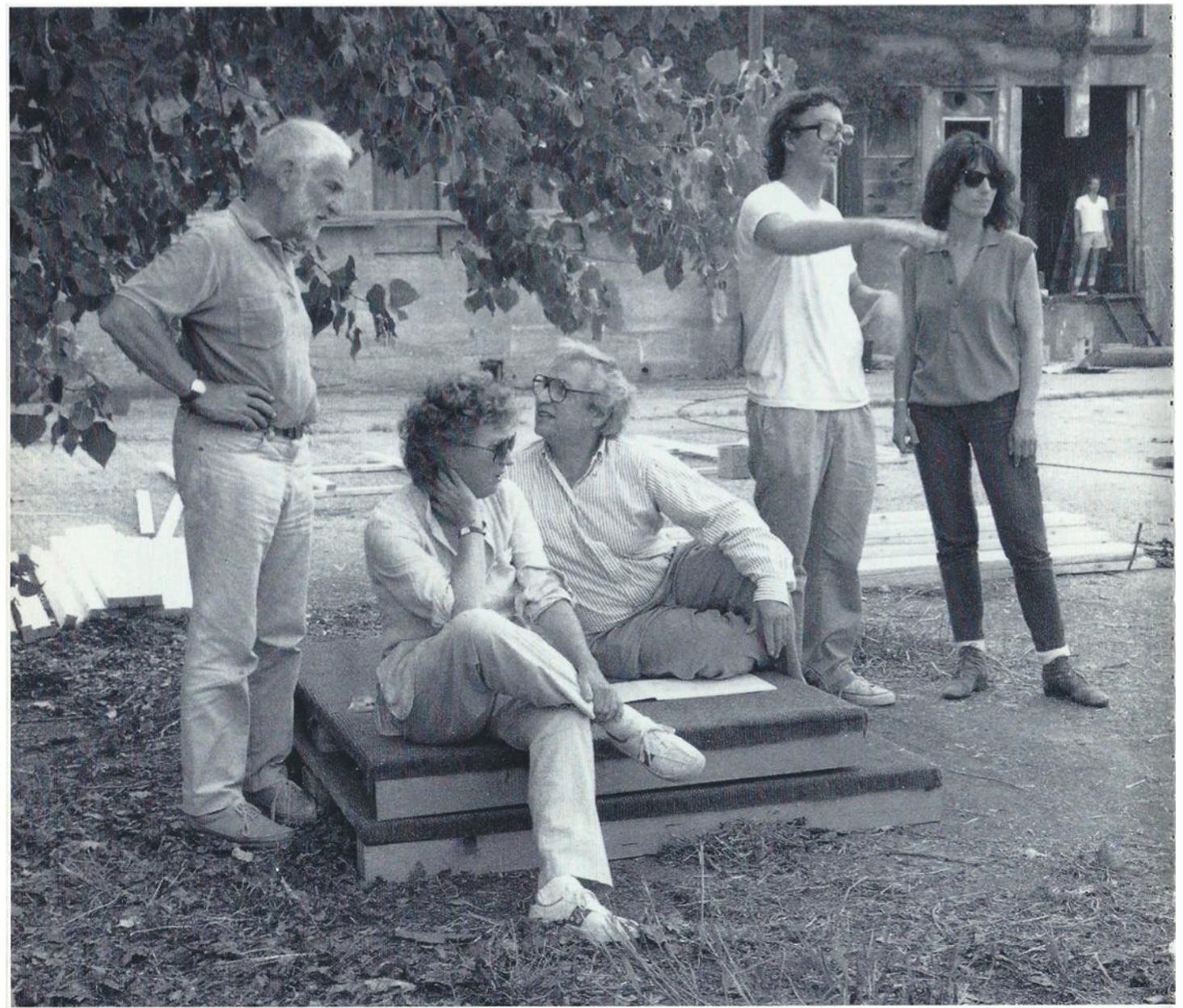

Figura 1 - Anthony Caro, Sheila Girling, Frank Gehry, Paul Lubowicki e Susan Nardulli durante a construção do projeto Sculpture Village, 1987 (@ Ferriel Waddington. Imagem gentilmente cedida por Jon Isherwood, a 5 de julho de 2020). 


\section{Sculpture village}

O projeto Sculpture Village resulta, assim, do trabalho realizado em 1987 no workshop organizado pelos Triangle Artists, uma iniciativa que, cinco anos depois da primeira edição tinha já conquistado fama a nível mundial. Acerca deste projeto em concreto, não existe, no entanto, muita informação, uma vez que a Sculpture Village, para além de ter sido praticamente ignorada pela crítica de época, foi desmantelada pouco tempo depois da sua conclusão.

Assim, a informação recolhida sobre este projeto baseia-se, fundamentalmente, no testemunho de alguns dos principais envolvidos e num conjunto de fotografias, depositadas no Frank O. Gehry papers. Series I. Architectural projects, parte do Getty Research Institute Special Collections ${ }^{4}$, que mostram o projeto depois de concluído. Nestas imagens, é evidente a atenção dada ao elemento escultórico e a utilização de resíduos e materiais reciclados, sendo também de assinalar o modo como rampas, escalas, nichos e fachadas conferem uma dimensão escultórica ao conjunto, dando origem, para todos os efeitos, a uma aldeia de esculturas. Será interessante determonos um pouco numa definição da palavra "aldeia":

"Em urbanística, grupo de habitações projetado de forma mais ou menos unitária por forma a constituir um conjunto edificado pensado organicamente como um bairro, para dar resposta às necessidades e aspirações de uma determinada categoria de habitantes."

Este é exatamente o objetivo do projeto proposto, sendo que a única diferença é o facto de responder às necessidades de uma categoria de objetos: os objetos escultóricos que compõem o conjunto.

Num depoimento contido no Anuário do workshop de 1987, o artista Anthony Caro escreveu:

"Frank Gehry, Jon Isherwood, and I met in New York in May and discussed the general avenue of approach. We decided to make architecture in much the same way as many of us make sculpture, moving parts into place and changing them, working loosely with a small team, all members contributing ideas and suggestions. Before the workshop, Jon enlarged six or seven of my sculptures and these became the pivots of our village structure. As soon as he saw them, Frank wanted to use them as they stood - expanding them into rooms, connected by walkways, using levels and bridges and creating a coherent environment. He incorporated sheet steel and tree trunks, creating a focus. Sheila Girling used interior spaces and wall spaces, painting wooden offcuts and collaging walls, ceilings and floors to accentuate the architecture; she also used coloured plexiglass in dark areas to create coloured light. Jon Isherwood built outdoor furniture, sculptural seating areas and tent-like roofs. Susan Nardulli and Paul Lubowicki built skeleton structures of fish and snake timbers, large enough to be walked into within the basically plywood village. Parick Hoystracht and his team of carpenters followed our lead imaginatively and creatively. The surprising thing was the closeness of vision with which we all saw the project and the degree of cooperation and the give and take with which we worked. Of course it wasn't architecture - nor sculpture, not painting either, but it was a step in the dark a crazy step pointing to what could be possible." 6 
O testemunho de Jon Isherwood vem confirmar-nos esta vontade de uma permanente e intensa colaboração entre os vários participantes, e acrescenta dados importantes acerca do modo como decorreu o trabalho. Para o já referido primeiro encontro em Nova lorque, e por forma poder perceber quais seriam as intenções de Gehry relativamente a este trabalho, Caro e Isherwood levaram uma maquete de uma escultura. Isherwood recorda, divertido, o momento em que, com Caro, teve de transportar pela cidade uma estátua de cerca de trinta quilos até chegar ao local do encontro. $\mathrm{O}$ objeto em questão, em forma de $\mathrm{T}$, tinha já um caráter arquitetónico, sugerindo ao espectador possibilidades de se movimentar através do espaço e de aceder, por meio de uma escada, a um segundo compartimento situado num nível acima.

Caro propôs de imediato transformar a maquete da escultura numa verdadeira "peça de arquitetura" e Gehry respondeu-lhe: "Tony, if you wanna turn it into architecture you just need to go ahead and do it!". 7 De facto, o desenvolvimento do trabalho ficou marcado por uma colaboração plena, não só entre os três artistas presentes nesta primeira reunião, mas também entre todos aqueles que participaram no workshop. 0 desafio lançado era o de dar prioridade à forma e realizar um projeto que integrasse os vários elementos propostos por cada um dos participantes. Depois deste primeiro encontro, cada um escolheu uma parte do projeto, após o que voltaram a reunir para, em conjunto, decidirem a escala e a dimensão dos elementos projetados, por forma a conferir fluidez e sincronia ao projeto e simplificar a sua realização e composição.

Tanto Caro como Isherwood tinham inicialmente pensado que o projeto seria composto por um único elemento, mas o trabalho acabou por resultar num conjunto de componentes que dava ao visitante a possibilidade de se movimentar: caminhando por entre os elementos, contornando o conjunto, trepando, ou subindo e descendo vários níveis. As duas referências centrais e confessas foram Antoni Gaudí mas também o Cubismo, pelo modo como este propõe múltiplas vistas de um único objeto, a partir de um mesmo ponto de observação. ${ }^{8}$

A abordagem ao projeto parece ter sido feita de um modo semelhante ao de um projeto de uma cidade. Uma das primeiras preocupações de Gehry foi, efetivamente, a de murar o conjunto uma vez que, tal como numa cidade, a ideia era existir um elemento de proteção e de fortificação que fosse também, em simultâneo, representativo da independência de uma comunidade. No interior, criavam-se as condições para o estabelecimento de uma relação entre os visitantes e as peças, baseada nas emoções. O diálogo entre os diversos elementos e os movimentos por estes sugeridos transformavam a aldeia numa espécie de parque de jogos para crianças. 


\section{estudoprévio}

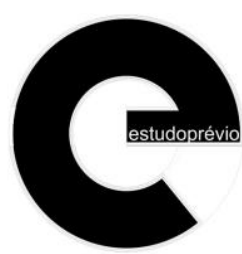

PT | P02 | EP18| w2021

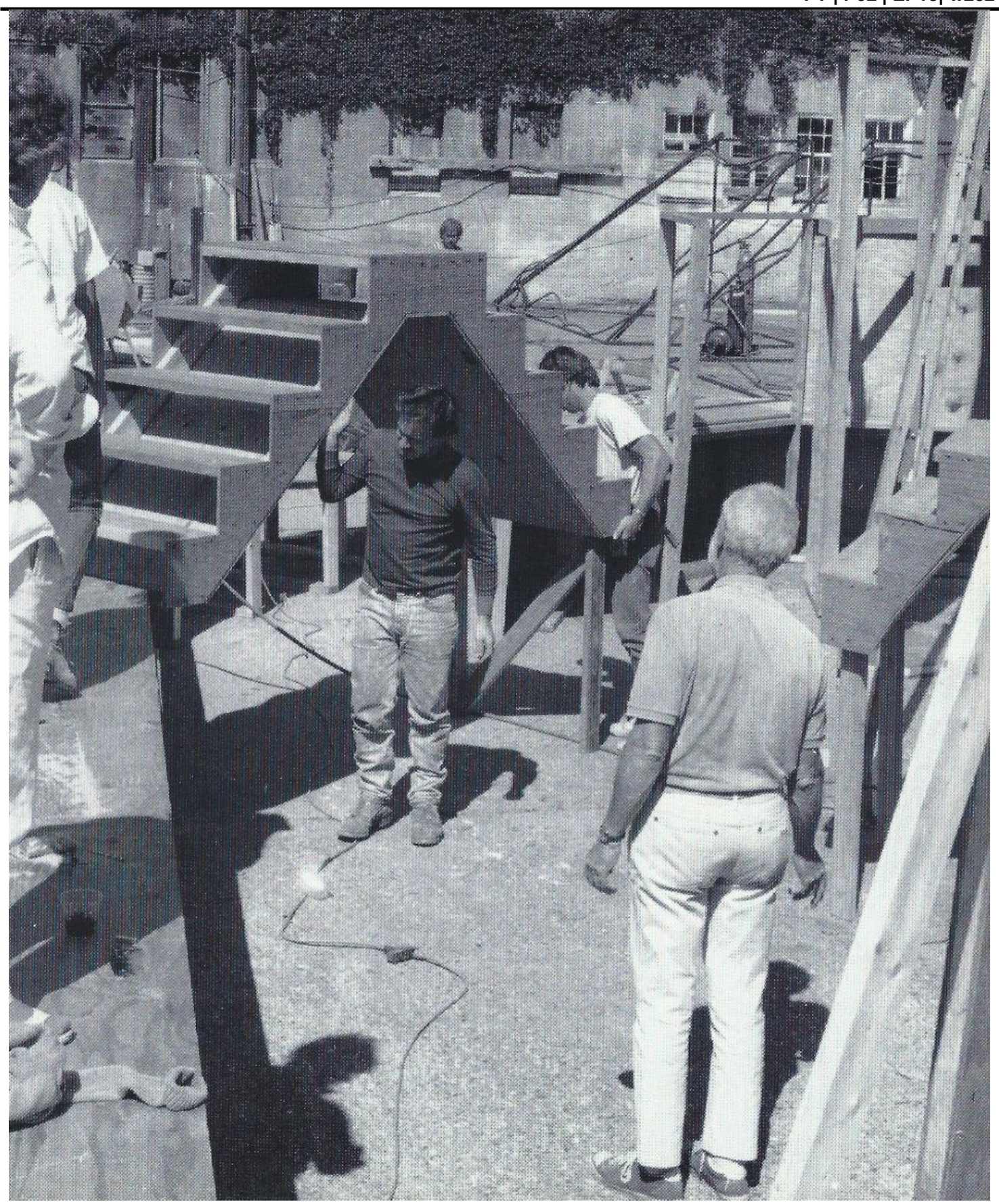

Figura 2 - Sculpture Village, construção, 1987 (@ Ferriel Waddington. Imagem gentilmente cedida por Jon Isherwood, a 5 de julho de 2020).

\section{Entre a escultura e a arquitetura}

Os autores definiram em mais de uma ocasião esta peça como uma fusão sinuosa, mas coerente, entre escultura e arquitetura, ou seja, uma Sculpitecture ${ }^{9}$, termo que 
encontramos no vocabulário da crítica de arte na referência ao trabalho de Anthony Caro. Segundo definição de Karen Wilkin, trata-se de uma "portmanteau word that has been attached to Anthony Caro's enterable structures" (Wilkin, 2002: 422) e o próprio Caro usava este termo com frequência, para se referir a alguns dos seus trabalhos.

A relação entre escultura e arquitetura foi um tema presente em permanência no trabalho de Caro, logo desde o início dos seus estudos de escultura na Royal Academy School de Londres. A arquitetura da Grécia, da Índia e do México constituíram grandes fontes de inspiração para o seu trabalho, assim como a Catedral de Chartres e, mais em particular, a obra de Gaudí em Barcelona, pelo modo como esta relacionava e combinava elementos escultóricos e elementos arquitetónicos. Mais que os detalhes arquitetónicos, interessam-lhe os volumes. Muitos dos trabalhos de Caro têm, de facto, um caráter arquitetónico, alguns mesmo com referências expressas ao universo da arquitetura. ${ }^{10}$ Esta sua atenção ao mundo da arquitetura está patente no projeto Village Sculpture e, tal como Gehry pode ser definido como um "arquiteto-escultor", também Caro foi um "escultor-arquiteto", sendo interessante notar como o ponto de união entre as duas figuras é, precisamente, a atenção e o interesse pelas duas artes que têm em comum.

Num texto escrito dois anos antes da realização deste projeto, Frank Gehry fazia referência ao papel da escultura no seu trabalho:

"If you try to understand my work on the basis of fugal order, structural integrity and formalized definitions of beauty, you are apt to be totally confused. I approach each building as a sculptural object, a spatial container [...] The manipulation of the inside of the container is for me an independent, sculptural problem and no less interesting than the design of the container itself." (Arnell, Bickford, 1985: 112)

Na sua procura por incorporar a beleza da escultura na edificação, Gehry deixa-se guiar pelos escultores, aprendendo com eles. A tridimensionalidade, os materiais e a modelação do espaço são alguns dos aspetos comuns às duas disciplinas e uma das questões fundamentais, não apenas neste trabalho, mas em toda a obra de Gehry. No entanto, segundo o próprio afirma, não foi fácil, ao início, cruzar a fronteira entre arquitetura e escultura, se é que essa fronteira realmente existe. O trabalho sobre a matéria na sua dimensão concreta será, provavelmente, o mais importante elo de ligação entre as obras produzidas pelos artistas e o trabalho de Frank Gehry. De facto, trata-se de um arquiteto que conhece a fundo os materiais e as suas propriedades $e$ que, de uma forma que é única, procede à sua transformação. Aço, vidro e pedra são assim entendidos como elementos plásticos que serão modelados com extrema delicadeza nos seus edifícios.

Terão as obras de arte influenciado as suas escolhas no que respeita aos materiais? Provavelmente sim, uma vez que é o próprio Gehry a afirmar que, para o projeto do Frederick R. Weisman Museum, se lembrou das esculturas de Ellsworth Kelly:

"By the time of the Weisman Museum, we could no longer use lead, copper outside so I went to stainless. I'd seen a number of Ellsworth Kelly's sandblasted stainless sculptures, and I loved them because they looked like suede. I was going to use it on the Weisman." (Friedman, 2002: 132)

Regressando à Sculpture Village, o facto de a obra ter passado desapercebida aos 
olhos da crítica deve-se muito provavelmente ao facto de a peça não ter chegado até nós, por ter sido demolida pouco tempo depois da sua conclusão. A Triangle Artists, fundada poucos anos antes, não dispunha de um espaço próprio para a realização do workshop, pelo que a peça foi construída num terreno pertencente a um privado. Para além disso, os recursos económicos de que dispunham eram limitados, o que levou a que a construção tenha decorrido num espaço de tempo curto e a que não houvesse qualquer tipo de manutenção das peças. Assim, expostas à intempérie, as peças acabaram, infelizmente, por ficar destruídas. Ainda foi equacionada a hipótese de recuperar os materiais e voltar a utilizar as esculturas, mas rapidamente se tornou claro que essa operação acarretava custos demasiado elevados, pelo que os artistas optaram por fazer réplicas em aço de algumas das peças e utilizá-las na criação de novas obras escultóricas ${ }^{11}$. De qualquer modo, a Sculpture Village era um projeto que tinha sido concebido em moldes experimentais e que nunca tinha sido encarada como algo definitivo, representando antes um desafio pessoal assumido pelas três principais figuras envolvidas, que decidiram pôr-se à prova por um período de tempo limitado.

Jon Isherwood acredita que o encontro com Frank Gehry marcou de forma profunda o seu percurso como artista, levando-o a investigar acerca de materiais pouco usuais e a sua manipulação. Recorda ainda, com apreço, um trabalho em que a colaboração se baseava na confiança, e em que cada um expressava a sua opinião, em pé de igualdade com os restantes. Um modo acertado de fazer a partilha do trabalho, tanto para a escultura, como para a arquitetura. ${ }^{12}$

O projeto da Sculpture Village vem demonstrar que, quando a arquitetura e a escultura se fundem, há lugar à criação de objetos únicos e originais. A escultura transforma-se, para Gehry, num laboratório de experimentação, e a sua arquitetura torna-se, por sua vez, numa referência e fonte de inspiração para os artistas que participaram no workshop, influenciando a natureza do seu trabalho e seus processos de criação, como se viu no caso de Jon Isherwood.

A relação entre a arquitetura e a escultura é revelada na sua evidência, assim como a capacidade de Gehry em transpor a ténue linha que as separa: mostrando que domina os processos de criação artística e dando vida a elementos arquitetónicos em permanente diálogo com o mundo da escultura.

\section{Agradecimentos}

Gostaríamos de dedicar umas breves, mas sinceras palavras de agradecimento a todos as pessoas que responderam às nossas questões e aos nossos pedidos, e que nos enviaram materiais e fotografias originais. Agradecemos muito em particular a Olivia Bax, da Barford Sculptures Ltd, Karen Wilkin e, finalmente, Jon Isherwood, que nos concedeu uma entrevista especial na qual nos falou deste projeto, da sua relação com Frank Gehry e de alguns interessantes episódios de bastidores. Foi para nós uma experiência incrível. Muito obrigado a todos. 


\section{Bibliografia}

Anthony Caro: teacher and student notes with key word cards. Tate Britain, 26 January - 17 April 2005. Disponível em: https://www.tate.org.uk/file/anthony-caroteachers-pack [Consult. em dezembro 2020].

ARNELL P.; BICKFORD T. - Frank Gehry buildings and projects. Rizzoli: New York, 1990. ISBN: 08478054339780847805433.

BREITSCHMID, Markus - Can architectural art-form be designed out of construction? Virginia: Architecture edition, 2004. ISBN: 978-0-9702-8208-8

BÖTTICHER, Karl - The principles of the Hellenic and Germanic ways of building with regard to their application to our present way of building. In FOGED, Isak Worre; HVEJSEL, Marie Frier - Reader: Tectonics in Architecture. Aalborg: Aalborg University Press, 2018.

CARO, Anthony [entrevista]. Depoimento recolhido por Tim Marlow, para o web site https://www.webofstories.com a 24 de janeiro de 2008.

Disponível em: https://www.webofstories.com/playAll/anthony.caro?sld=15931 [Consult. em junho 2020].

FRIEDMAN, M. - Art and Objecthood: Essays and Reviews. Chicago: University of Chicago Press, 1998. ISBN: 9780226263199.

Frank Gehry teaches design and architecture (masterclass). www.masterclass.com Disponível em: https://www.masterclass.com/classes/frank-gehry-teachesdesign-and-architecture [Consult. em junho 2020]

GEHRY, Frank O. - Project 1987-NA: Caro/Gehry Collaboration: "Sculpture Village": Reference images. In Frank O. Gehry papers. Series I. Architectural projects. Getty Research Institute Special Collections. Disponível em: http://hdl.handle.net/10020/2017m66_eafe834e5285658cdf34f6d2e93a5db3 [Consult. em maio 2020].

GUASH A. M.; ZULAIKA J. - Learning from the Bilbao Guggenheim The Museum as a Cultural Tool. Reno (Nevada): Center for Basque Studies - University of Nevada, 2005. ISBN: 1877802506.

KRAUSS, R. E. - Sculpture in the Expanded Field. The Originality of The Avant-Garde and Other Modernist Myths. Cambridge, Massachusetts: The MIT Press, 1985. ISBN: 0262610469.

MacCORMAC, R. - When art meets architecture: Anthony Caro retrospective. Tate etc, $\mathrm{N} . .5$ (setembro 2005). Disponível em: https://www.tate.org.uk/tate-etc/issue-5autumn-2005/when-art-meets-architecture [Consult. em julho 2020].

PEABODY, R. - Anglo-American Exchange in Postwar Sculpture 1945 - 1975. Los Angeles: Getty publications, 2011. ISBN 978-1-60606-069-8.

POLLACK, Sydney - Sketches of Frank Gehry [documentário]. Los Angeles: Mirage, 2006. (83 min). Disponível (versão em italiano) em: http://www.rai.it/dl/RaiTV/programmi/media/Contentltem-ae3eeeaf-8e3b-4c398b82-8656776a2de9-cinema.html

SPENCE, R. - Anthony Caro: The shaping of a radical. Financial Times (24 julho 2015). Disponível em: https://www.ft.com/content/f46d8d34-2ef8-11e5-91aca5e17d9b4cff [Consult. em julho 2020].

STAN, A. - From Object to Field. AD (Architectural Design). Vol. 67, N.ㄴ 5, 6. May, June 1997, p. 24-31.

STAN, A. - Minimalism: Sculpture and Architecture. Art \& Design. Special Issue (1997). London, p. 22-30. ISSN: 0267-3991.

Triangle Artists' Workshop. www.triangleartsnyc.org Disponível em 
PT | P02 | EP18| w2021

https://www.triangleartsnyc.org/workshop [Consult. em junho 2020]

WILKIN, Karen - At the galleries. Partisan Review, Vol. 69, №. 3 (Estate 2002). p. 422433.

Disponível

em: http://www.bu.edu/partisanreview/books/PR2002V69N3/HTML/files/assets/basic$\mathrm{html} /$ index.html\#367 [Consult. em dezembro 2020].

\footnotetext{
1 Ver, sobre este assunto: https://www.triangleartsnyc.org/history [Consult. em 2020].

2 Ver, sobre este assunto: TAYLOR, Kate - Creating Together. The Sun (25 setembro 2007). Disponível em: https://www.nysun.com/arts/creating-together/63332/.

3 Informações fornecidas por Karen Wilkin, num e-mail enviado a 2 de julho de 2020. Historiadora, curadora, crítica de arte, Karen Wilkin tem um percurso profissional estreitamente ligado à Triangle Artists desde a sua fundação.

${ }^{4}$ Todas as polaroids do projeto Sculpture Village estão digitalizadas e disponíveis através do link: https://rosettaapp.getty.edu/delivery/DeliveryManagerServlet?dps_pid=IE3878245

${ }^{5}$ In "In urbanistica, gruppo di abitazioni progettato più o meno unitariamente in modo da costituire un complesso edilizio studiato organicamente come un quartiere urbano, per rispondere ai bisogni o ai desiderî di una data categoria di abitanti". In Villagio. Instituto della Encicolpedia Italiana, www.treccani.it/ [Consult. em dezembro 2020]. Disponível em: https://www.treccani.it/vocabolario/villaggio/

${ }^{6}$ Excerto do depoimento de Anthony Caro no Anuário do Workshop da Triangle Artists de 1987, editado em setembro de 1987, fornecido por Karen Wilkin, em e-mail enviado a 2 de julho de 2020.

${ }^{7}$ Excerto da entrevista realizada pelos autores a Jon Isherwood a 10 de julho de 2020.

8 ibidem

9 ibidem

10 TATE BRITAIN - Anthony Caro: teacher and student notes with key word cards. Disponível online: https://www.tate.org.uk/file/anthony-caro-teachers-pack [Consult. em dezembro 2020].

11 Idem nota 7.

12 Idem.
} 